\title{
Comparing the Therapeutic Effects of Aloe vera and Olive Oil Combination Cream versus Topical Betamethasone for Atopic Dermatitis: A Randomized Double-blind Clinical Trial
}

\author{
Yunes Panahi ${ }^{1}$, Nassim Rastgar ${ }^{2}$, Ali Zamani ${ }^{1}$, Amirhossein Sahebkar $^{3,4,5,6}$ * \\ ${ }^{1}$ Pharmacotherapy Department, Faculty of Pharmacy, Baqiyatallah University of Medical Sciences, Tehran, Iran \\ ${ }^{2}$ Department of Pharmaceutics, Faculty of Pharmacy, Pharmaceutical Science Branch, Islamic Azad University, Tehran, Iran \\ ${ }^{3}$ Halal Research Center of IRI, FDA, Tehran, Iran \\ ${ }^{4}$ Neurogenic Inflammation Research Center, Mashhad University of Medical Sciences, Mashhad, Iran \\ ${ }^{5}$ Biotechnology Research Center, Pharmaceutical Technology Institute, Mashhad University of Medical Sciences, Mashhad, Iran \\ ${ }^{6}$ Polish Mother's Memorial Hospital Research Institute (PMMHRI), Lodz, Poland
}

Received March 28, 2018

Reviewed July 31, 2020

Accepted August 28, 2020

\section{* Correspondence}

Amirhossein Sahebkar

Biotechnology Research Center, Pharmaceutical Technology Institute, Mashhad University of Medical Sciences, Mashhad, Iran

Tel: +98-511-800-2288

E-mail: sahebkara@mums.ac.ir, amirssaheb2000@yahoo.com
Objectives: Atopic dermatitis (AD) is a prevalent and chronic, pruritic inflammatory skin condition that can influence all age groups. $A D$ is associated with a poor health-related quality of life. This randomized clinical trial was performed to compare the effectiveness of Olivederma (combination of aloe vera and virgin olive oil) or betamethasone regarding disease severity, quality of life, serum IgE and eosinophil count.

Methods: Thirty-six AD patients were randomly allocated to topical Olivederma or betamethasone, and were followed for 6 weeks.

Results: Total SCORAD severity scores showed significant decrease in both groups, while it was more prominent in Olivederma group (64.5\% improvement in Olivederma vs. $13.5 \%$ improvement in Betamethasone, p-value < 0.001). Quality of life (DLQI questionnaire) of AD patients was significantly improved after 6 weeks treatment with Betamethasone $(22.3 \%, p<0.001)$ and Olivederma (60.7\%, p-value < 0.001). Olivederma group showed a significantly lower DLQI score in comparison with Betamethasone treated patients after 6 weeks of therapy $(p<0.001)$. Improvements in eosinophil count and serum IgE was observed.

Conclusion: In summary, this study shows that Olivederma is superior to topical Betamethasone after 6 weeks of therapy with regard to disease severity, quality of life and eosinophil count.

Keywords: atopic dermatitis, Aloe vera, olive oil, corticosteroid, topical administration

\section{INTRODUCTION}

Atopic dermatitis (AD) is a common chronic inflammatory genetically determined disease of the skin which is marked by increased ability to form regain (IgE), with increased susceptibility to allergic rhinitis and asthma, and hereditary disposition to a lowered threshold for pruritus. It affects up to $20 \%$ of children and $1 \%$ to $3 \%$ of adults [1]. AD is associated with a decrement in health-related quality of life [2].
Topical medications are the mainstay of AD treatment. Even in more severe cases needing systemic or phototherapy, they are often used in combination with these interventions [3]. Topical corticosteroids have been used to treat $\mathrm{AD}$ for more than 60 years [4]. The effectiveness of topical steroids is demonstrated $[3,4]$.

Topical corticosteroids exert various cutaneous side effects includng purpura, telangiectasia, striae, focal hypertrichosis, and acneiform or rosacea-like eruptions [5]. Skin atrophy is our 
greatest concern, which can be induced by any TCS, though high-potent steroids are more hazardous $[4,5]$. Due to these adverse reactions and considering the fact that $\mathrm{AD}$ patients may have multiple flares during their life, it is quite necessary to develop no-steroidal anti-inflammatory and disease modifying agents.

Olivederma is a combination of virgin olive oil (VOO) and aloe vera gel. $\mathrm{VOO}$ has $82 \%$ unsaturated fatty acids [6], which helps in moisturizing dryness and removing Staphylococcus aureus from colonized AD skin [6]. Moreover, the anti-inflammatory activity of aloe vera has been demonstrated [7].

There are few randomized clinical trials for comparing the effects of topical corticosteroids and natural topical products like Olivederma on the severity of $\mathrm{AD}$ and the quality of life of patients. Therefore, we conducted this randomized clinical trial for this purpose.

\section{MATERIALS AND METHODS}

\section{Trial design}

This randomized clinical trial was performed during July 2009-July 2010 in Dermatology clinics of Tehran Baqiyatallah University. Patients satisfying inclusion criteria were randomly allocated in two groups receiving Olivederma or betamethasone, in a parallel group design (1:1 ratio) using block randomization (with a block size of 4). The specialists and staffs were blinded through trial.

\section{Participants and study setting}

We enrolled 36 patients with atopic dermatitis for this randomized, double-blind placebo- clinical trial. Patients were diagnosed by the U.K. Working Party's Diagnostic Criteria for atopic dermatitis [8].

Inclusion criteria were defined as: age OF 17-70 years, confirmed diagnosis of atopic dermatitis, not having allergy to herbal products, and providing the written informed consent. These patients were excluded: patients who do not show adherence to intervention (based on weekly follow-up calls; defined as refusing to consume utmost $10 \%$ of recommended treatments), and those with any adverse reactions to the medications.

Ethical Committee of Tehran Islamic Azad University approved the study.

\section{Interventions}

Intervention group received Olivederma topical (Kimi Daru Pharmaceutical Co, Tehran, Iran) for 2 times a day for 6 weeks and the control group received topical betamethasone (Kimi Daru Pharmaceutical Co, Tehran, Iran) 2 times a day for 6 weeks.

\section{Randomization}

We used permuted block randomization sequences (created using Microsoft ${ }^{\mathrm{TM}}$ Excel version 2010 software; the block sizes were considered fixed as 4). A blinded statistician generated the sequentially numbered containers for concealment. The blinded secretary of outpatient dermatology clinic enrolled participants using sealed sequential numbers with attached pockets containing medications (identical betamethasone and Olivederma topical medications). Blinded dermatologists of clinic explained the interventions to the participants and checked their adherence by weekly calls and monthly visits. All adverse events were recorded by them.

\section{Sample size}

This primary aim of this study was to compare the effects of topical Olivederma and betamethasone on SCORAD severity index of atopic dermatitis. Moreover, the quality of life (DLQI), serum IgE and eosinophil count in the peripheral blood were also compared. The sample size was obtained regarding the primary outcome of SCORAD scores. The effect size was selected as 15 scores mean difference between intervention groups ( $\mathrm{SD}=$ 15). Also 5\% type I error and $80 \%$ power were considered.

Formula written here was used:

$$
n=\left(Z_{1-\frac{\alpha}{2}}+Z_{1-\beta}\right)^{2} / d \text { where } d=\left(D_{1}-D_{2}\right) /(\sigma \sqrt{2})
$$

Considering 5\% loss to follow-up, sample size per each group was determined as 17 atopic dermatitis patients.

\section{Outcomes and measurements}

The primary outcome variables were SCORAD severity index of atopic dermatitis [9]. Moreover, the validated Persian version of Dermatology Life Quality Index (DLQI) questionnaire was measured for assessing the quality of life.

Blood samples of patients were obtained $(10 \mathrm{cc})$ to measure 
the serum IgE and peripheral blood eosinophil count.

\section{Statistical methods}

Quantitative data are shown as mean \pm SD. Within-group (before/after intervention) differences of were tested by paired t-test. Between-groups differences are analyzed by Independent $\mathrm{t}$-test and the time ${ }^{*}$ intervention interaction is analyzed by repeated measures AMOVA. Categorical data are reported as numbers (percentages) and were tested by $\chi^{2}$, Fisher's exact, or Monte Carlo tests; where indicated. p-values less than 0.05 were considered statistically significant.

\section{RESULTS}

79 patients with $\mathrm{AD}$ were recruited for initial assessment of eligibility. Twenty-four patients were excluded due to not meeting the inclusion criteria and 19 ones refused to participate. The remaining 36 atopic dermatitis patients were randomly allocated to topical Olivederma or betamethasone, and were followed for 6 weeks.

The studied AD patients had a Mean (Range) age of 33.41 (13-57) years. They were $56 \%$ male. We did not observe significant differences for demographic characteristics and past medical history between intervention groups (Table 1). Overall, 36 T2DM patients (19 in the Olivederma group; 16 patients in the Betamethasone group) successfully finished the trial and were analyzed.

Regarding the severity of AD (Table 2), total SCORAD

Table 1. Baseline characteristics of patients with atopic dermatitis randomly allocated in topical Betamethasone and Olivederma interventions

\begin{tabular}{lccc}
\hline \multirow{2}{*}{$\begin{array}{c}\text { Patients' } \\
\text { characteristics }\end{array}$} & \multicolumn{2}{c}{ Group } & Difference \\
\cline { 2 - 3 } Age (years) & Betamethasone & Olivederma & p-value \\
\hline Sex & $30.44 \pm 9.65$ & $36.37 \pm 12.45$ & 0.123 \\
\hline Male & $6(35.3 \%)$ & $10(52.6 \%)$ & 0.296 \\
\hline Female & $11(64.7 \%)$ & $9(47.4 \%)$ & \\
Allergic rhinitis & $4(23.5 \%)$ & $8(42.1 \%)$ & 0.238 \\
$\begin{array}{l}\text { Asthma and allergic } \\
\text { rhinitis }\end{array}$ & $13(76.47 \%)$ & $9(47.4 \%)$ & 0.074 \\
$\begin{array}{l}\text { Positive family } \\
\text { history of atopic } \\
\text { dermatitis }\end{array}$ & $17(100 \%)$ & $16(84.2 \%)$ & 0.087 \\
\hline
\end{tabular}

scores showed significant decrease in both groups, while it was more prominent in Olivederma group (64.5\% improvement in Olivederma vs. 13.5\% improvement in Betamethasone, p-value $<0.001)$.

More interestingly, we observed that quality of life (DLQI questionnaire, Table 3) of AD patients was significantly improved after 6 weeks treatment with Betamethasone (22.3\%, p $<0.001)$ and Olivederma $(60.7 \%$, p-value $<0.001)$. Olivederma group showed a significantly lower DLQI score in comparison with Betamethasone treated patients after 6 weeks of therapy ( $p$ $<0.001)$.

We found that eosinophil count (Table 4) of Betamethasone group was significantly increased $(p=0.011)$, while it was significantly decreased after 6 weeks of therapy with Olivederma $(36 \%, \mathrm{p}=0.003)$.

We observed that Olivederma did significantly decrease serum IgE level (Table 4), while it was remained constant in Betamethasone group. However, the between-group difference of Betamethasone and Olivederma was non-significant.

\section{DISCUSSION}

In this randomized, double blind clinical trial we observed that topical Olivederma is superior to topical Betamethasone after 6 weeks of therapy with regard to disease severity, quality of life and eosinophil count of $\mathrm{AD}$ patients.

Table 2. Comparing topical Betamethasone and Olivederma on SCORAD severity index of patients with atopic dermatitis randomly allocated in interventions

\begin{tabular}{clccc}
\hline Indices & Phase & Betamethasone & Olivederma & p-value* \\
\hline SCORAD- A & Baseline & $44.4 \pm 8.7$ & $75.1 \pm 7.2$ & $<0.001$ \\
& 6th week & $35.5 \pm 9.9$ & $23.3 \pm 13.6$ & \\
& p-value & 0.006 & $<0.001$ & \\
SCORAD- B & Baseline & $9.6 \pm 1.5$ & $9.4 \pm 1.7$ & $<0.001$ \\
& 6th week & $8.1 \pm 2.3$ & $3.2 \pm 1.8$ & \\
& p-value & 0.004 & $<0.001$ & \\
SCORAD- C & Baseline & $15.8 \pm 2.7$ & $19.5 \pm 0.9$ & $<0.001$ \\
& 6th week & $15.2 \pm 3.4$ & $8.2 \pm 5.7$ & \\
& p-value & 0.545 & $<0.001$ & \\
SCORAD & Baseline $_{\text {total }}$ & $58.4 \pm 7.3$ & $67.3 \pm 6.3$ & $<0.001$ \\
& 6th week $^{*} 50.5 \pm 10.7$ & $23.9 \pm 13.3$ & \\
& p-value & 0.003 & $<0.001$ & \\
\hline
\end{tabular}

"Within-group (time effect) significance.

*Between-group (intervention * time effect) significance. 
Yunes Panahi, et al.

Table 3. Comparing topical Betamethasone and Olivederma on quality of life (DLQI scores) of patients with atopic dermatitis randomly allocated in interventions

\begin{tabular}{clccc}
\hline Indices & \multicolumn{1}{c}{ Phase } & Betamethasone & Olivederma & p-value* \\
\hline DLQI & Baseline & $22 \pm 3.2$ & $18.3 \pm 2.8<0.001$ \\
score & 6th week & $17.1 \pm 5.4$ & $7.2 \pm 1.4$ & $<$ \\
& p-value $^{\#}$ & $<0.001$ & $<0.001$ & \\
DLQI & Excellent improve $_{\text {change }}$ & $0(0 \%)$ & $6(31.6 \%)<0.001$ \\
& Mild improve & $7(41.2 \%)$ & $13(68.4 \%)$ \\
& No changes & $9(52.9 \%)$ & $0(0 \%)$ \\
& Exacerbated & $1(5.9 \%)$ & $0(0 \%)$ \\
\hline
\end{tabular}

"Within-group (time effect) significance.

*Between-group (intervention * time effect) significance.

Traditionally, topical corticosteroids are pivotal therapeutic agents for patients with $\mathrm{AD}$ [3-5]. Considering the chronic nature of $\mathrm{AD}$, its multiple recurrences throughout life, and the disadvantageous effects of topical corticosteroid; it is warranted to conduct studies aimed to develop non-steroidal agents and with minimal harms [6]. Natural products have always been a potential resource to discover and develop drugs. Combinations of such products have been introduced in literature [3] to conduct therapeutic effects. However, the evidence is insufficient to support the superiority of such natural products for treatment of chronic inflammatory dermatitis like AD. We conduct this study to compare the effectiveness of Olivederma (combination of aloe vera and virgin olive oil) and Betamethasone for improving the quality of life of $\mathrm{AD}$ patients and decreasing the severity of disease.

Several studies support the role of Aloe vera in wound healing [10-19]. It has been found to decrease the healing time of burn wound patients in comparison with petroleum gel [18]. Its effects have been postulated to be exerted through its antiinflammatory effects by prostaglandins and bradykinin $[7,14$, $16,17]$. However, aloe vera was not better that placebo in the treatment of slight to moderate psoriasis vulgaris [20]. To the best of our knowledge, there were no randomized controlled trials comparing the efficacy of aloe vera and betamethasone in treatment of $\mathrm{AD}$ patients. We observed that Olivederma (combination of aloe vera and virgin olive oil) has significant effects in improving the severity of $\mathrm{AD}$ and the quality of life of patients.

Emollients are a standard of care for inhibition of dryness, steroid-sparing effect, and maintenance therapy in $\operatorname{AD}[4,6]$. Virgin olive oil coat the skin, occluding and protecting it by
Table 4. Comparing topical Betamethasone and Olivederma on eosinophil count and serum IgE level of patients with atopic dermatitis randomly allocated in interventions

\begin{tabular}{clccc}
\hline \multicolumn{1}{c}{ Indices } & Phase & Betamethasone & Olivederma & p-value* \\
\hline Eosinophil & Baseline & $1.7 \pm 2.4$ & $2.03 \pm 1.66$ & $<0.01$ \\
count & 6th week & $1.79 \pm 2$ & $1.3 \pm 1.16$ & \\
& p-value & 0.011 & 0.003 & \\
\multirow{2}{*}{ Serum IgE } & Baseline & $332.3 \pm 75.6$ & $358.1 \pm 51.6$ & 0.676 \\
& 6th week & $326.9 \pm 93.3$ & $339.9 \pm 56.2$ & \\
& p-value & 0.23 & 0.007 & \\
\hline
\end{tabular}

"Within-group (time effect) significance.

*Between-group (intervention * time effect) significance.

slowing down transepidermal water loss and increasing hydration within the stratum corneum $[6,21]$. Also, it makes the skin look less rough and scaly [21]. The superiority of Olivederma in comparison with Betamethasone regarding the severity of dryness reported by our patients might be due to such moisturizing effects of olive oil.

$\mathrm{AD}$ patients have a decreased quality of life [2]. Evidence reveals that they have inferior mental health scores compared with diabetes or hypertension patients, and inferior social functioning scores compared with hypertension patients. In comparison with psoriasis patients, $\mathrm{AD}$ patients are reported to have inferior scores in the role-physical, vitality, social functioning, role-emotional, and mental health SF-36 domains [2]. In this study we observed that all of patients treated with topical Olivederma showed improvements in DLQI scores, while only $53 \%$ of $\mathrm{AD}$ patients treated with Betamethasone have mild improvements regarding the quality of life.

The association between the severity of $\mathrm{AD}$ symptoms and serum IgE level has been observed [22, 23]. Also, to our knowledge, the disease severity and personal or family history of respiratory atopy are associated with higher eosinophil count [24]. We found that both eosinophil count and serum IgE level are significantly decreased after 6 weeks of therapy with Olivederma, however eosinophil count of Betamethasone treated patients were found to be increased. Moreover, betamethasone did not show significant effects on serum IgE. Now, we do not know the exact cause of such advantageous effects of Olivederma in comparison with Betamethasone. Further studies are required to confirm this finding.

This study had some limitations, as we could not follow our patients for long-term and examine the effectiveness of Olivederma in severe flare-ups. However, we showed the superiority 
of Olivederma to Betamethasone by various subjective and objective markers in short-term. The considerable improvement of $\mathrm{AD}$ patients after treatment with a natural combination of Virgin olive oil and aloe vera is quite encouraging to conduct more trials investigating its superiority to other topical regimens especially corticosteroids.

In conclusion, this randomized double-blind clinical trial shows that Olivederma, a combination of aloe vera and Virgin olive oil, is superior to topical Betamethasone after 6 weeks of therapy with regard to disease severity, quality of life and eosinophil count. Further clinical trials are required.

\section{ACKNOWLEDGMENT}

The authors would like to thank Kimi Daru Pharmaceutical Co. for providing the studied drugs. We are also thankful to the "Clinical Research Development Unit of the Baqiyatallah Hospital" for providing technical support.

\section{CONFLICT OF INTEREST}

We declare no conflicting financial or non-financial interests.

\section{ORCID}

Yunes Panahi, https://orcid.org/0000-0002-2504-8356

Nassim Rastgar, https://orcid.org/0000-0002-4634-957X

Ali Zamani, https://orcid.org/0000-0002-7826-6867

Amirhossein Sahebkar, https://orcid.org/0000-0002-8656-1444

\section{REFERENCES}

1. Bae JM, Choi YY, Park CO, Chung KY, Lee KH. Efficacy of allergen-specific immunotherapy for atopic dermatitis: a systematic review and meta-analysis of randomized controlled trials. J Allergy Clin Immunol. 2013;132(1):110-7.

2. Kiebert G, Sorensen SV, Revicki D, Fagan SC, Doyle JJ, Cohen J, et al. Atopic dermatitis is associated with a decrement in healthrelated quality of life. Int J Dermatol. 2002;41(3):151-8.

3. Eichenfield LF, Tom WL, Berger TG, Krol A, Paller AS, Schwarzenberger K, et al. Guidelines of care for the management of atopic dermatitis: section 2. Management and treatment of atopic dermatitis with topical therapies. J Am Acad Dermatol. 2014; 71(1):116-32

4. Hoare C, Li Wan Po A, Williams H. Systematic review of treat- ments for atopic eczema. Health Technol Assess. 2000;4(37):1191.

5. Callen J, Chamlin S, Eichenfield LF, Ellis C, Girardi M, Goldfarb $\mathrm{M}$, et al. A systematic review of the safety of topical therapies for atopic dermatitis. Br J Dermatol. 2007;156(2):203-21.

6. Verallo-Rowell VM, Dillague KM, Syah-Tjundawan BS. Novel antibacterial and emollient effects of coconut and virgin olive oils in adult atopic dermatitis. Dermatitis. 2008;19(6):308-15.

7. Vázquez B, Avila G, Segura D, Escalante B. Antiinflammatory activity of extracts from Aloe vera gel. J Ethnopharmacol. 1996; 55(1):69-75.

8. Williams HC, Burney PG, Hay RJ, Archer CB, Shipley MJ, Hunter JJ, et al. The U.K. working party's diagnostic criteria for atopic dermatitis. I. Derivation of a minimum set of discriminators for atopic dermatitis. Br J Dermatol. 1994;131(3):383-96.

9. Schallreuter K, Levenig C, Berger J. Severity scoring of atopic dermatitis: the SCORAD index. Consensus report of the European task force on atopic dermatitis. Dermatology. 1993;186(1): 23-31.

10. Atiba A, Ueno H, Uzuka Y. The effect of aloe vera oral administration on cutaneous wound healing in type 2 diabetic rats. J Vet Med Sci. 2011;73(5):583-9.

11. Chithra P, Sajithlal GB, Chandrakasan G. Influence of aloe vera on the healing of dermal wounds in diabetic rats. J Ethnopharmacol. 1998;59(3):195-201.

12. Chithra P, Sajithlal GB, Chandrakasan G. Influence of Aloe vera on the glycosaminoglycans in the matrix of healing dermal wounds in rats. J Ethnopharmacol. 1998;59(3):179-86.

13. Jafarzadeh H, Arabi M, Najafi N, Ahadi AM. Effect of aloe vera gel on TGF- $\beta$ gene expression in incisional skin wound in BALB/c mice. J Gorgan Univ Med Sci. 2014;16(3):16-23.

14. Maenthaisong R, Chaiyakunapruk N, Niruntraporn S, Kongkaew $C$. The efficacy of aloe vera used for burn wound healing: a systematic review. Burns. 2007;33(6):713-8.

15. Molazem Z, Mohseni F, Younesi M, Keshavarzi S. Aloe vera gel and cesarean wound healing; a randomized controlled clinical trial. Glob J Health Sci. 2014;7(1):203-9.

16. Takzare N, Hosseini MJ, Hasanzadeh G, Mortazavi H, Takzare A, Habibi P. Influence of Aloe Vera gel on dermal wound healing process in rat. Toxicol Mech Methods. 2009;19(1):73-7.

17. Takzaree N, Hassanzadeh G, Rouini MR, Keshtkar A, Manayi A, Haajiakhondi A. Histological study of wound repair with topical aloe vera gel in rat. Tehran Univ Med J. 2015;73(9):660-7.

18. Visuthikosol V, Chowchuen B, Sukwanarat Y, Sriurairatana S, Boonpucknavig V. Effect of aloe vera gel to healing of burn wound a clinical and histologic study. J Med Assoc Thai. 1995; 78(8):403-9.

19. Yadav KCH, Kumar JR, Basha SI, Deshmukh GR, Gujjula R, 
Yunes Panahi, et al.

Santhamma B. Wound healing activity of topical application of aloe vera gel in experimental animal models. Int J Pharm Bio Sci. 2012;3(2):63-72.

20. Paulsen E, Korsholm L, Brandrup F. A double-blind, placebocontrolled study of a commercial Aloe vera gel in the treatment of slight to moderate psoriasis vulgaris. J Eur Acad Dermatol Venereol. 2005;19(3):326-31.

21. Viola P, Viola M. Virgin olive oil as a fundamental nutritional component and skin protector. Clin Dermatol. 2009;27(2):159-
65.

22. Clendenning WE, Clack WE, Ogawa M, Ishizaka K. Serum IgE studies in atopic dermatitis. J Invest Dermatol. 1973;61(4):233-6.

23. Johnson EE, Irons JS, Patterson R, Roberts M. Serum IgE concentration in atopic dermatitis. Relationship to severity of disease and presence of atopic respiratory disease. J Allergy Clin Immunol. 1974;54(2):94-9.

24. Uehara M, Izukura R, Sawai T. Blood eosinophilia in atopic dermatitis. Clin Exp Dermatol. 1990;15(4):264-6. 